\title{
GESTAÇÃO DIANTE DA PANDEMIA DE COVID-19 - AS PRINCIPAIS REPERCUSSÕES PSICOLÓGICAS NEGATIVAS E SUAS CAUSAS: UMA REVISÃO INTEGRATIVA
}

Renata Cabral Rodrigues Feitosa 1, ORCID ID 0000-0003-4150-5825; Flávia Thalia Guedes Farias², ORCID ID 00000-0003-1586-5667; Livia Dantas Fragoso², ORCID ID 0000-0002-7436-7660; Vilma Raquel Lima Ramalho de Holanda², ORCID ID 0000-0003-0700-4971; Ludmila Cavalcante Agra3; ORCID ID 0000-0002-1467-2137; Cristina Ruan Ferreira de Araújo ${ }^{4}$, ORCID ID 0000-0002-6611-7290

\section{FILIAÇÃO}

(1) Universidade Federal de Campina Grande, Discente de Medicina

(2) Centro Universitário de Patos UNIFIP, Discente de Medicina

(3) Universidade Federal de Campina Grande, Discente de Medicina

(4) Universidade Federal de Campina Grande, Docente

\section{AUTOR CORRESPONDENTE}

Renata Cabral Rodrigues Feitosa; renatacrfeitosa@gmail.com; Rua Reinaldo Tavares de Melo, № 25; apto 201, Campina Grande-PB; Universidade Federal de Campina Grande.

\section{MENSAGENS-CHAVE}

A COVID-19 gerou mudanças no mundo, provocando alterações da rotina social de toda a população.

O grupo de mulheres grávidas que lida com mudanças fisiológicas físicas e psicológicas resultantes do processo gestacional.

O isolamento social e o desconhecimento dos impactos vivenciados por gestantes propiciaram impactos psicológicos relevantes.

Foi verificado ansiedade, depressão, estresse, ideação suicida, insônia,Transtorno do Estresse Pós-traumático (TEPT), entre outras. Como achado destaca-se a convergência das mudanças sociais como causadoras de desequilíbrios psicológicos em gestantes.

\section{RESUMO}

INTRODUÇÃO: A pandemia da COVID-19 impactou o mundo em diversas esferas, propiciando consequências na vida dos indivíduos. Nesse sentido, a população de gestantes sofreu com as mudanças mundiais propostas pela situação pandêmica, corroborando em repercussões psicológicas determinantes na qualidade de vida materna. OBJETIVO: Reunir e descrever os impactos psicológicos negativos da pandemia em mulheres grávidas,destacando as suas causas relacionadas. MÉTODO: Trata-se de uma revisão integrativa da literatura realizada através da busca de artigos nas plataformas virtuais Medline e LILACS. A pesquisa foi executada utilizando os termos MeSH "Pregnant Women", "COVID-19" e"coronavirus infection"; e os termos DeCS "Gestantes", "COVID-19". Foram selecionados estudos completos disponíveis online, em língua inglesa, espanhola ou portuguesa e publicados nos últimos dois anos. RESULTADOS: Dos 194 artigos analisados, 21 preencheram os critérios de inclusão, sendo a maioria estudos transversais $(n=16)$, que oferece ao tema uma boa base observacional. Categorias de repercussões psicológicas foram identificadas como ansiedade, depressão, estresse, entre outros. Causas associadas: Mudanças e dificuldades no acesso à assistência em saúde no pré-natal, parto e pós-natal, baixa rede de apoio e aumento do conflito em casa, medo de auto infecção ou infecção do feto e complicações na gravidez. DISCUSSÃO: Partindo dos resultados, percebeu-se que tal contexto da COVID-19 proporcionou sérios problemas psicológicos nas gestantes, principalmente a ansiedade e a depressão, devido aos perfis sociodemográficos, ao excessivo acesso à informações e às mídias sociais, às mudanças nos comportamentos saudáveis e às dificuldades em se prestar assistência em saúde às grávidas. CONCLUSÃO: A pandemia causada pela COVID-19 potencializa as vulnerabilidades da gestação, favorecendo implicações negativas na saúde mental das gestantes. Assim, necessita-se de remodelação nos serviços e ações em saúde, 
visando corrigir as falhas no oferecimento e continuidade do atendimento, atendendo às particularidades de cada paciente, frente às adversidades geradas pela pandemia.

\section{PALAVRAS-CHAVE: Mulheres Grávidas; Infecção por Coronavírus; COVID-19; Saúde Mental}

\section{ABSTRACT}

INTRODUCTION: The pandemic of COVID-19 impacted the world in several spheres, causing consequences in the lives of individuals. In this sense, the population of pregnant women suffered with the global changes proposed by the pandemic situation, corroborating in determinant psychological repercussions on the quality of maternal life. OBJECTIVE: To gather and describe the negative psychological impacts of the pandemic on pregnant women, highlighting their related causes. METHOD: This is an integrative literature review carried out by searching for articles on the Medline and LILACS virtual platforms. The search was performed using the MeSH terms "Pregnant Women", "COVID-19" and "coronavirus infection"; and the DeCS terms "Pregnant Women", "COVID-19". Full studies available online, in English, Spanish or Portuguese language and published within the last two years were selected. RESULTS: Of the 194 articles analyzed, 21 met the inclusion criteria, with the majority being cross-sectional studies $(n=16)$, which offers the topic a good observational basis. Categories of psychological repercussions were identified as anxiety, depression, stress, among others. Associated causes: Changes and difficulties in access to health care in prenatal, delivery, and postnatal care, low support network and increased conflict at home, fear of self-infection or infection of the fetus, and pregnancy complications. DISCUSSION: Based on the results, it was perceived that such context of COVID-19 provided serious psychological problems in pregnant women, especially anxiety and depression, due to sociodemographic profiles, excessive access to information and social media, changes in healthy behaviors, and difficulties in providing health care to pregnant women. CONCLUSION: The pandemic caused by COVID-19 potentiates the vulnerabilities of pregnancy, favoring negative implications on the mental health of pregnant women. Thus, it is necessary to remodel the health services and actions, aiming to correct the flaws in the offer and continuity of care, attending to the particularities of each patient, facing the adversities generated by the pandemic.

\section{KEYWORDS: Pregnant Women; Coronavirus Infection; COVID-19; Mental Health}

\section{INTRODUÇÃO}

Desde o início do ano de 2020, a pandemia da Coronavírus Disease 2019 (COVID-19), que afetou o planeta de maneiras desiguais, impactou todo o funcionamento logístico, orgânico e social de todos os indivíduos, corroborando em impactos negativos que perdurarão por um longo tempo. A infecção do novo coronavírus influência de forma negativa a rotina de todas as pessoas, e apresenta-se como um problema de saúde pública mundial que se agrava em virtude da lentidão com que a vacina está chegando à população, principalmente em países subdesenvolvidos, bem como as restrições de estudo no desenvolvimento delas, já que os grupos controle foram específicos, a citar testagem em adultos e idosos saudáveis $(1,2)$.

Partindo desse contexto pandêmico, destaca-se a vivência da gravidez, a qual é um processo fisiológico desejado por milhares de mulheres em todo o mundo, em que se criam expectativas, desejos, sonhos e inseguranças. Nesse sentido, observou-se que, diante da pandemia e do consequente isolamento social, houveram mudanças comportamentais significativas nesse grupo de mulheres. Essa realidade é algo preocupante, tendo em vista que, segundo a Organização Mundial de Saúde (OMS), as gestantes necessitam de cuidados especiais e uma assistência mais detalhada em virtude de possíveis complicações gestacionais, o que não está sendo viabilizado diante dos impasses gerados pela COVID-19 (3).

Nesse viés, é necessário destacar as adversidades advindas da pandemia da COVID-19, as quais partem desde a infecção viral propriamente dita, aos efeitos indiretos decorrentes da prevenção, como a vivência do isolamento social que, por seguinte, gerou uma mudança nas modalidades de sociabilidade, trabalho e atendimentos médicos, que encontram-se à distância e com menor recorrência.4 Nas gestantes, percebeu-se problemas relacionados a esse contexto pandêmico, como o distanciamento dessas com seus familiares e amigos, bem como a dificuldade em manter a rotina pré-natal que envolve o processo gestacional (5).

Essa situação se converge em impactos negativos de cunho físico e social, destacando-se que, no âmbito físico e orgânico, verificou-se um aumento de peso, desenvolvimento de maus hábitos alimentares, ausência de atividade física e redução do tempo de sono (2). Já com relação à esfera psicológica, em que a pandemia repercute em quadros ansiosos desenvolvidos na ausência de convívio social, seja esse sentimento relacionado ao medo de contrair a doença, a falta de familiares e amigos próximos, a insegurança sobre gestar em um período de incertezas e, até mesmo, o autoquestionamento sobre a capacidade de cuidar de uma criança, o que se verificou em estudos randomizados que analisaram essa patologia (6). Além da ansiedade, agravações psicológicas como Transtorno de Estresse Pós-traumático (TEPT), Transtorno Obsessivo Compulsivo (TOC), insônia, Depressão Pós-parto, foram verificadas em centenas de mulheres, explicitando como a pandemia afetou um momento, que já denota inseguranças, tão esperado e desejado, corroborando em um quadro clínico perigoso em um grupo que já apresenta mais riscos $(3,7)$.

Dessa forma, uma análise mais detalhada das repercussões psicológicas diante da pandemia da COVID-19 em gestantes é essencial para entender que a situação mundial influi além das repercussões diretas do vírus, sendo estas determinantes no futuro materno e do recém-nascido, devendo-se, portanto, desvendar esses efeitos e, assim, promover um cuidado voltado para a problemática. Logo, este estudo tem como objetivo identificar e explicar os impactos psicológicos negativos da pandemia em mulheres grávidas, destacando as suas causas relacionadas.

\section{METODOLOGIA}


O estudo trata-se de uma revisão integrativa da literatura construída em etapas esquematizadas de acordo com o proposto por Botelho, Cunha e Macedo (2011). Essa organização foi realizada da seguinte forma: pesquisa pela temática e formulação da questão norteadora; definição das estratégias de busca, dos descritores e das bases de dados; estabelecimento dos critérios de exclusão e inclusão de artigos e organização dos artigos pré-selecionados; seleção duplo-cega de artigos, em um primeiro momento, a partir da leitura dos títulos e resumos; posteriormente foi realizada a leitura na íntegra e análise crítica dos artigos relevantes; categorização dos artigos selecionados; interpretação das informações coletadas e síntese dos achados para discussão (8).

A busca foi realizada a partir da questão norteadora elaborada segundo a estratégia PECO. A partir desse instrumento definiu-se como pergunta: "Quais as repercussões psicológicas diante da pandemia de COVID19 em gestantes e suas causas?"

\begin{tabular}{|c|c|}
\hline P (população) & Gestantes \\
\hline E (exposição) & Pandemia de COVID-19 \\
\hline C (comparador) & Não-exposição \\
\hline O (desfecho) & Impactos psicológicos \\
\hline
\end{tabular}

Quadro 1: Pergunta estruturada para a análise das evidências (PECO). (Fonte: autoria própria)

A pesquisa foi executada por dois pesquisadores e de forma padronizada nas plataformas virtuais Medical Literature Analysis and Retrieval System Online (MEDLINE) e Literatura Latino-Americana e do Caribe em Ciências da Saúde (LILACS). Os descritores em saúde foram determinados conforme as especificidades das bases de dados utilizadas. Por isso, para o MEDLINE optou-se pelos termos MeSH (Medical Subject Headings) e para o LILACS foram aplicados os termos DeCS (Descritores em Ciências da Saúde). Neste último preferiu-se a versão na língua inglesa para ampliar os resultados Dessa forma, os termos utilizados foram "Pregnant Women", "COVID-19" e "coronavirus infection", que possuem correspondência nos dois formatos de descritores em saúde. Em seguida, a estratégia de busca foi organizada por meio do cruzamento dos descritores com o auxílio dos operadores booleanos "AND" e "OR", da seguinte forma: "Pregnant Women" AND "COVID-19" OR "coronavirus infection". Salienta-se que optou-se por não incluir um descritor específico para distúrbios psicológicos a fim de ampliar os achados relacionados com gestantes durante o período de pandemia.

Os critérios de inclusão estabelecidos foram: artigos em fulltext (disponibilizado completo online), em língua inglesa, espanhola ou portuguesa e publicados entre dezembro de 2019 e abril de 2021. De acordo com os critérios de exclusão foram descartados estudos indisponíveis online, revisões, editoriais, artigos de opinião, arquivos que se caracterizassem como "projeto piloto" e não apresentassem desfecho.

Dessa forma, os artigos identificados como elegíveis passaram, primeiramente, por uma análise de títulos e resumos que permitiu a exclusão dos trabalhos em divergência com os objetivos e critérios de inclusão (Figura 1). Após isso, foi efetuada a leitura dos artigos selecionados de forma crítica e integral, com categorização e síntese das informações quanto ao nome dos autores, ano de publicação, abordagem metodológica, nível de evidência, objetivos, principais resultados e observações, como apresentado na Tabela 1. A avaliação do nível de evidência por tipo de estudo dos trabalhos seguiu a metodologia proposta no protocolo "Oxford Centre for Evidence-based Medicine (March 2009)" em sua atualização de 2009, que possui sua estrutura detalhada em uma escala com dez níveis de evidências (9).

\section{RESULTADOS}

Dessa forma, encontrou-se um total de 194 artigos elegíveis

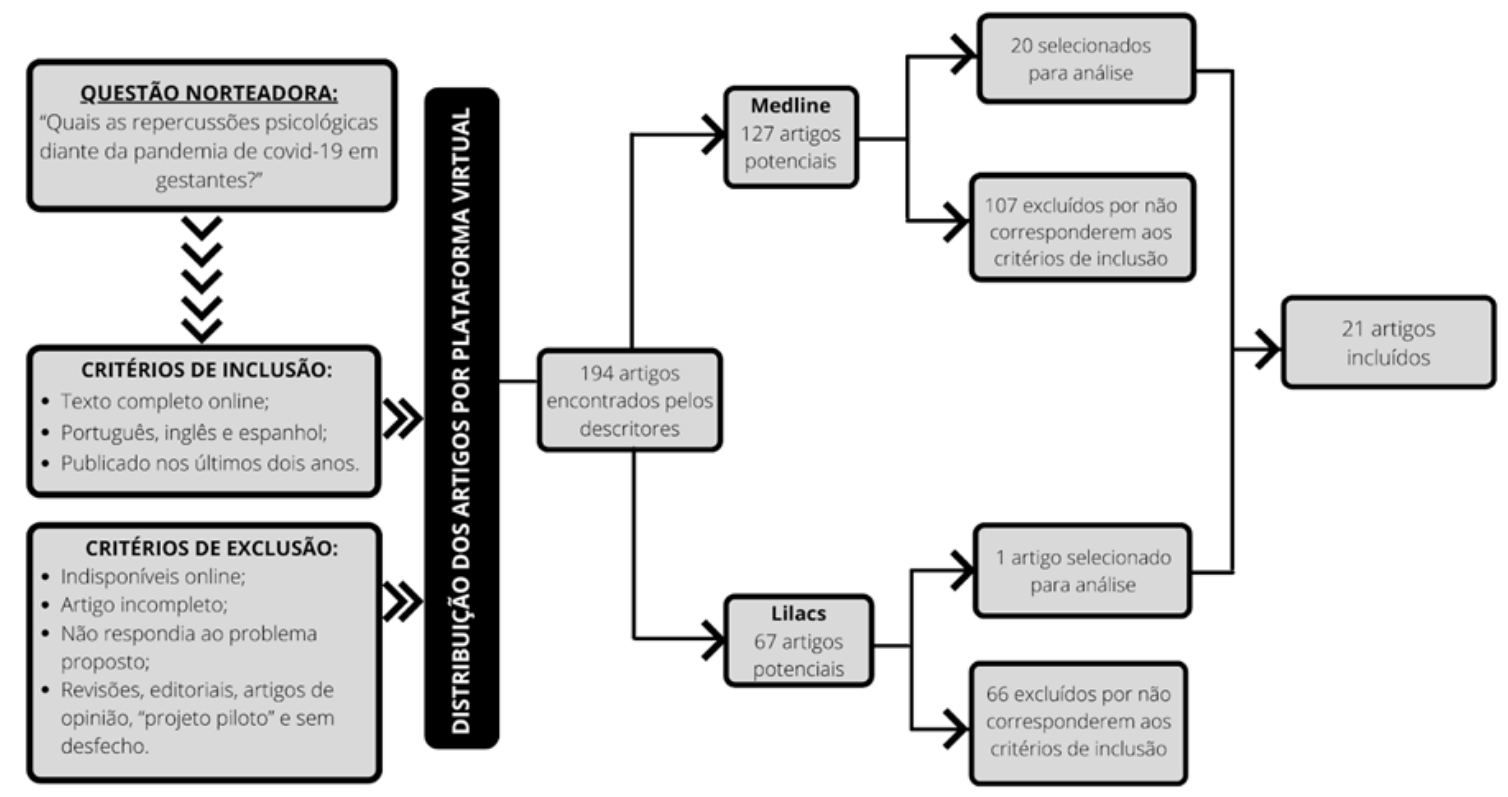

Figura 1: Busca e seleção de artigos. (Fonte: autoria própria) 
para análise de títulos e resumos. Após essa avaliação, foram selecionados 21 artigos (Figura 1). Dos artigos incluídos, $61 \%$ foram publicados em 2020 , sendo o restante publicado em 2021, seis foram conduzidos no Irã $(4,5,7,10$ $12)$, cinco na China $(1,2,13-15)$, dois na Turquia $(16,17)$ e nos EUA $(6,18)$ e um no Brasil (3), Canadá (19), Reino Unido (20), Polônia (21), Singapura (22) e Itália (23). O método predominante foi o estudo transversal ( $n=16)$, sendo os outros métodos um estudo de coorte prospectivo (2), um relato de experiência fenomenológico descritivo (11) e um estudo de caso-controle e transversal (17); o predomínio do estudo transversal pode estar relacionado aos seus atributos fundamentais, os quais oferecem bases para o entendimento de um determinado efeito estudado em uma população específica por meio da observação da frequência de ocorrência ou alteração de um evento. A maioria dos artigos continham amostras pequenas, tendo apenas $5(13,14,18-20)$ mais de mil participantes. Em termos de qualidade da evidência dos artigos incluídos, apenas quatro artigos $(2,14,19,20)$ foram identificados como 2B e, portanto, com bom nível de evidência por se tratarem de estudos de coorte ou ensaios clínicos randomizados com metodologia bem delineada e amostra populacional ampla. Desse modo, a maioria dos estudos $(\mathrm{N}=14)$ foram caracterizados como nível 3B $(1,4-7,10,12$, $13,15-18,21,22)$ e três artigos foram qualificados como 4 (3, 11, 23), por apresentarem fragilidades metodológicas, amostra limitada ou se caracterizar como relato de experiência.

Quinze estudos pesquisaram ansiedade, tendo treze encontrado níveis elevados de ansiedade em gestantes (4, 5, 7,11-14,16-19,21,22). Quanto aos outros dois artigos, Zhou et al. (2020) (1) descobriu, ao comparar gestantes e não gestantes, que mulheres não grávidas apresentavam mais sintomas psiquiátricos (depressão, ansiedade, desconforto físico, insônia, transtorno do estresse pós traumático) do que as gestantes; e Dong et al. (2021) (15) encontrou que o nível de ansiedade das gestantes permaneceu o mesmo quando comparado com os de gestantes pré-pandêmicas. Shayganfard et al. (2020) (4) evidenciou que maior gravidade de sintomas ansiosos levaram ao adiamento ou cancelamento de consultas médicas de rotina das gestantes. Apenas um artigo investigou especificamente repercussões em gestantes de risco (17).

Ademais, encontrou-se como repercussão psicológica depressão $(7,10,13-15,19,22,23)$, estresse $(4,11,20,21)$, Transtorno do estresse pós-traumático $(1,16,23)$ e pensamento de automutilação (14).

Dos fatores relacionados às repercussões psiquiátricas, mostraram-se agentes protetores a prática de exercícios físicos $(5,14,19)$, boas práticas de cuidado em relação a COVID-19 (7) e boa rede de apoio (19); acesso à informação e às mídias sociais se mostrou fator protetor quando se havia acesso a informações confiáveis, de contenção da pandemia e notícias positivas $(1,2,15)$ e fator agravante quando não confiáveis, noticiavam aumento dos casos e em uso excessivo $(2,14,22)$; Alguns artigos dedicaram-se a investigar as percepções e experiências das gestantes $(3,11)$ onde encontraram comportamentos de resiliência como a criação de grupos virtuais de apoio e de aulas de preparação para o parto; interessantemente 5 encontrou que a felicidade ocasionada pela gestação causa efeito benéfico para a saúde materna e pode ser usada como estratégia para a diminuição da ansiedade.

No que se refere aos principais fatores estressores foram elencados: mudanças e dificuldades no acesso à assistência em saúde no pré-natal, parto e pós-natal (18-20), baixa rede de apoio e aumento do conflito em casa $(6,11,18,21)$, medo de autoinfecção ou do infecção do feto $(4,10,11,18)$ e complicações na gravidez $(5,13,16,17,21)$.

\section{DISCUSSÃO}

A gestação é um período marcado por mudanças, sejam elas físicas, mentais e/ou sociais. Adentrando esse marco muito importante para uma mulher em um contexto de pandemia, percebe-se que há alterações significativas de uma estrutura social, marcada por medidas que visam a precaução de contágio, como o isolamento social, afastamento do trabalho, falta de informações sobre a morbidade e mortalidade em mulheres associada à possibilidade da transmissão vertical e, assim, instalando-se um ambiente facilitador de desenvolvimento de doenças mentais. Mediante os artigos revisados, foram vistos que 21 estudos abordavam diretamente o reflexo de tal contexto na gravidez, provocando sequelas psíquicas para a gestante. Fatores causais relacionados aos perfis sociodemográficos, ao acesso à informação e às mídias sociais, às mudanças nos comportamentos saudáveis, e à assistência em saúde: prénatal, parto e pós-natal foram elementos determinantes no desenvolvimento de repercussões psicológicas nas gestantes $(5,19,20)$.

A partir dos estudos analisados, verificou-se que existem perfis sociodemográficos, os quais contribuem para o mau prognóstico da evolução gestacional. Dentre eles, notou-se que gestantes que vivem em países emergentes ou subdesenvolvidos são mais propensas a manifestar problemas psicológicos. Isso acontece, uma vez que, por muitas dessas mulheres possuírem baixa renda e os maridos apresentarem empregos instáveis, elas se preocupam mais com as finanças e com a situação econômica familiar, desenvolvendo quadros de ansiedade (6). A exemplo do Brasil, o qual ranqueia na primeira posição entre as nações mais ansiosas e em seguida, o México, de acordo com o levantamento do "Tracking the Coronavirus", realizado pela empresa Ipsos em 2021 (16).

Além da condição econômica, percebeu-se que o estado civil e o nível educacional são importantes fatores predisponentes para maiores scores de depressão e de ansiedade. Isso se justifica, pois as mães solteiras se preocupam mais por não terem o apoio de um parceiro para ajudar a enfrentar as dificuldades do processo gestacional, as quais são agravadas pelo isolamento social e pela falta de suporte familiar $(10,21)$. Além disso, quanto maior o grau de escolaridade da grávida, maior é o risco de desenvolvimento de estresse a curto e médio prazo, posto que elas apresentam uma melhor literacia 


\begin{tabular}{|c|c|c|c|c|c|c|}
\hline $\begin{array}{c}\text { Autor/An } \\
0\end{array}$ & $\begin{array}{c}\text { Abordagem } \\
\text { metodológic } \\
\text { a }\end{array}$ & $\begin{array}{c}\text { Nível de } \\
\text { evidênci } \\
\text { a }\end{array}$ & Amostra & Objetivos & Resultados & Observações \\
\hline $\begin{array}{c}\text { Sinaci et } \\
\text { al }(2020)^{17}\end{array}$ & $\begin{array}{c}\text { Estudo } \\
\text { Transversal } \\
\text { de Caso- } \\
\text { Controle }\end{array}$ & $3 B$ & $\begin{array}{c}446 \\
\text { gestantes } \\
(246 \\
\text { gestantes } \\
\text { de alto risco } \\
200 \\
\text { gestantes } \\
\text { sem fatores } \\
\text { de risco) }\end{array}$ & \begin{tabular}{|c|} 
Analisar o nível \\
de ansiedade \\
de gestantes \\
com e sem \\
fatores de \\
risco, durante a \\
pandemia de \\
COVID-19.
\end{tabular} & $\begin{array}{c}\text { Houve aumento da } \\
\text { prevalência de ansiedade } \\
\text { em gestante de alto risco } \\
\text { quando comparadas com } \\
\text { gestantes normais. Entre } \\
\text { as gestantes de alto risco } \\
\text { fatores como } \\
\text { hospitalização prévia } \\
\text { aumentavam } \\
\text { significativamente o } \\
\text { escore de ansiedade. }\end{array}$ & $\begin{array}{l}\text { O estudo realizado em } \\
\text { hospital de cuidado para } \\
\text { pacientes com COVID-19, } \\
\text { ademais gestantes de alto } \\
\text { risco necessitam de maior } \\
\text { número de visitas ao } \\
\text { hospital, desse modo, esse } \\
\text { fatores podem ser vieses no } \\
\text { estudo que contribuíram } \\
\text { para o aumento da } \\
\text { ansiedade desse grupo } \\
\text { quando comparado ao } \\
\text { grupo controle. }\end{array}$ \\
\hline $\begin{array}{c}\text { Moyer et } \\
\text { al }(2020)^{18}\end{array}$ & $\begin{array}{l}\text { Estudo } \\
\text { transversal }\end{array}$ & $3 B$ & $\begin{array}{c}2740 \\
\text { gestantes }\end{array}$ & $\begin{array}{l}\text { Explorar o } \\
\text { impacto da } \\
\text { pandemia de } \\
\text { COVID-19 na } \\
\text { ansiedade de } \\
\text { gestantes e } \\
\text { identificar } \\
\text { fatores } \\
\text { associados. }\end{array}$ & $\begin{array}{c}\text { Encontrou-se aumento } \\
\text { nos níveis de ansiedade, } \\
\text { sendo o escore de } 20,6 \\
\text { pré-COVID e } 23,9 \text { durante } \\
\text { a pandemia. Fatores } \\
\text { relacionados encontrados: } \\
\text { interromper as consultas } \\
\text { pré-natais presenciais e } \\
\text { alterar o plano de parto do } \\
\text { parto hospitalar, aumento } \\
\text { do conflito em casa, medo } \\
\text { de ser infectado. }\end{array}$ & $\begin{array}{l}\text { Um viés deste estudo é a } \\
\text { confiança na avaliação } \\
\text { retrospectiva das mulheres } \\
\text { sobre ansiedade } \\
\text { relacionada a gravidez } \\
\text { antes da pandemia em } \\
\text { comparação com o quão } \\
\text { ansiosas estavam no } \\
\text { momento da pesquisa. } \\
\text { Além disso, o estudo } \\
\text { abrangeu mulheres com } \\
\text { acesso à tecnologia, uma } \\
\text { vez que a pesquisa foi } \\
\text { realizada online. }\end{array}$ \\
\hline $\begin{array}{c}\text { Shayganfa } \\
\text { rd et al } \\
(2020)^{4}\end{array}$ & $\begin{array}{l}\text { Estudo } \\
\text { Transversal }\end{array}$ & $3 B$ & $\begin{array}{c}103 \\
\text { mulheres } \\
\quad 66 \\
\text { gestantes e } \\
37 \text { mulheres } \\
\text { em até 6 } \\
\text { semanas } \\
\text { após o } \\
\text { parto) }\end{array}$ & \begin{tabular}{|c|} 
Investigar se a \\
ansiedade, o \\
estresse ou a \\
depressão em \\
relação à \\
saúde levaram \\
ao adiamento \\
ou \\
cancelamento \\
de consultas \\
médicas de \\
rotina.
\end{tabular} & $\begin{array}{c}\text { Evidenciou-se que maior } \\
\text { ansiedade em relação à } \\
\text { saúde e menores escores } \\
\text { de estresse previram o } \\
\text { adiamento ou } \\
\text { cancelamento das } \\
\text { consultas; Além disso, } \\
\text { níveis elevados de } \\
\text { ansiedade estavam } \\
\text { relacionados à } \\
\text { proximidade com pessoas } \\
\text { infectadas e ao medo de } \\
\text { se infectar. }\end{array}$ & $\begin{array}{l}\text { Não foi perguntado se } \\
\text { usaram estratégias } \\
\text { alternativas como a } \\
\text { consulta online. Mulheres } \\
\text { em fase pós-parto e com } \\
\text { mais filhos relataram maior } \\
\text { estresse. }\end{array}$ \\
\hline $\begin{array}{l}\mathrm{Ng} \text { et al } \\
(2020)^{22}\end{array}$ & $\begin{array}{l}\text { Estudo } \\
\text { Transversal }\end{array}$ & $3 B$ & $\begin{array}{c}324 \\
\text { gestantes }\end{array}$ & \begin{tabular}{|c|} 
Avaliar o \\
conhecimento \\
das gestantes \\
sobre COVID- \\
19 e suas \\
percepções do \\
impacto da \\
pandemia \\
sobre o \\
psicológico e a \\
gravidez.
\end{tabular} & $\begin{array}{c}\text { Parte significativa das } \\
\text { gestantes desconhecia ou } \\
\text { associava a infecção por } \\
\text { COVID-19 durante a } \\
\text { gravidez com sofrimento } \\
\text { fetal }(82,1 \%), \text { morte } \\
\text { intrauterina }(71,3 \%), \\
\text { anomalias fetais }(69,8 \%), \\
\text { abortos espontâneos } \\
(64,8 \%), \text { parto prematuro } \\
(67,9 \%) \text { e ruptura de } \\
\text { membranas }(61,4 \%) .\end{array}$ & $\begin{array}{l}116 \text { mulheres tiveram } \\
\text { triagem positiva para } \\
\text { ansiedade, } 59 \text { para } \\
\text { depressão e } 36 \text { para } \\
\text { estresse. Observou-se que } \\
\text { a falta de informações } \\
\text { oportunas e confiáveis } \\
\text { sobre a Covid-19 na } \\
\text { gravidez resulta em níveis } \\
\text { aumentados de depressão, } \\
\text { ansiedade e estresse. }\end{array}$ \\
\hline
\end{tabular}




\begin{tabular}{|c|c|c|c|c|c|c|}
\hline $\begin{array}{c}\text { Mortazavi; } \\
\text { Ghardashi } \\
(2021)^{11}\end{array}$ & $\begin{array}{c}\text { Fenomenológi } \\
\text { co } \\
\text { (pesquisa } \\
\text { qualitativa) }\end{array}$ & 4 & $\begin{array}{c}19 \\
\text { gestantes }\end{array}$ & $\begin{array}{l}\text { Compreender } \\
\text { as experiências } \\
\text { vividas por } \\
\text { mulheres } \\
\text { grávidas } \\
\text { durante a } \\
\text { epidemia de } \\
\text { COVID-19. }\end{array}$ & $\begin{array}{c}\text { Observou-se intenso } \\
\text { estresse, ansiedade e } \\
\text { depressão nas primeiras } \\
\text { semanas após o anúncio } \\
\text { oficial da pandemia no Irã. } \\
\text { Mostrou-se, ainda, } \\
\text { estratégias adaptativas } \\
\text { como criação de grupos } \\
\text { online de apoio e aulas } \\
\text { virtuais preparatórias para } \\
\text { o parto. }\end{array}$ & $\begin{array}{c}\text { A principal preocupação das } \\
\text { entrevistadas foi a } \\
\text { possibilidade de serem } \\
\text { infectados e por } \\
\text { consequência infectar o } \\
\text { feto. }\end{array}$ \\
\hline $\begin{array}{l}\text { Hamzehga } \\
\text { rdeshi et al } \\
(2021)^{7}\end{array}$ & $\begin{array}{c}\text { Estudo } \\
\text { Transversal }\end{array}$ & $3 B$ & $\begin{array}{c}318 \\
\text { gestantes }\end{array}$ & $\begin{array}{c}\text { Avaliar a } \\
\text { ansiedade } \\
\text { relacionada a } \\
\text { gravidez (ARG) } \\
\text { e seus fatores } \\
\text { associados } \\
\text { durante a } \\
\text { pandemia de } \\
\text { COVID-19. }\end{array}$ & $\begin{array}{c}21 \% \text { das gestantes tinham } \\
\text { ARG. } 42,1 \% \text { depressão e } \\
4,4 \% \text { ansiedade } \\
\text { relacionada ao COVID- } 19 . \\
\text { Observou-se também que } \\
\text { depressão e ansiedade } \\
\text { relacionada ao COVID-19 } \\
\text { aumentaram as chances } \\
\text { de ARG, respectivamente } \\
\text { em quatro vezes e } 13 \% .\end{array}$ & $\begin{array}{l}\text { Boas práticas em relação ao } \\
\text { COVID-19 diminuem as } \\
\text { chances de ARG em } 62 \% \text {. } \\
\text { Foi apontado como viés a } \\
\text { coleta de dados baseada } \\
\text { (em sua maioria) na web, o } \\
\text { que pode selecionar } \\
\text { pessoas com melhor status } \\
\text { socioeconômico. }\end{array}$ \\
\hline $\begin{array}{l}\text { Wu et al } \\
(2020)^{14}\end{array}$ & $\begin{array}{c}\text { Estudo } \\
\text { Transversal }\end{array}$ & $2 \mathrm{~B}$ & $\begin{array}{c}4124 \\
\text { gestantes } \\
\text { (2839 } \\
\text { avaliadas } \\
\text { antes da } \\
\text { declaração } \\
\text { pública de } \\
\text { pandemia } \\
\text { (grupo } \\
\text { 1)1285 } \\
\text { após } \\
\text { declaração } \\
\text { pública } \\
\text { (grupo2)) }\end{array}$ & $\begin{array}{l}\text { Examinar o } \\
\text { impacto do } \\
\text { surto de } \\
\text { COVID-19 na } \\
\text { prevalência de } \\
\text { depressão e } \\
\text { ansiedade e os } \\
\text { fatores de risco } \\
\text { correspondente } \\
\text { s a gravidez. }\end{array}$ & $\begin{array}{l}\text { Observou-se, no grupo } 2 \text {, } \\
\text { taxas maiores de sintomas } \\
\text { depressivos ( } 26,0 \% \text { vs } \\
29,6 \% \text {,) e de ansiedade } \\
\text { do que no grupo } 1.0 \\
\text { grupo } 2 \text { também era mais } \\
\text { propenso a pensamentos } \\
\text { de automutilação. As } \\
\text { maiores taxas de } \\
\text { depressão foram } \\
\text { associadas ao número de } \\
\text { casos recém-confirmados } \\
\text { COVID-19, infecções } \\
\text { suspeitas e mortes por } \\
\text { dia. }\end{array}$ & $\begin{array}{c}\text { As características } \\
\text { demográficas foram } \\
\text { semelhantes entre os dois } \\
\text { grupos do estudo; Fatores } \\
\text { maior risco de depressão: } \\
\text { ter menos de educação } \\
\text { universitária, ter uma renda } \\
\text { familiar anual baixa, } \\
\text { trabalhar meio período ou } \\
\text { menos, ser filha único, } \\
\text { suporte familiar pobre, } \\
\text { menos de } 7 \text { horas por } \\
\text { semana de exercícios } \\
\text { físicos e ter uma placenta } \\
\text { prévia. }\end{array}$ \\
\hline $\begin{array}{l}\text { Lebel et al } \\
(2020)^{19}\end{array}$ & $\begin{array}{c}\text { Estudo } \\
\text { Randomizado }\end{array}$ & $2 \mathrm{~B}$ & $\begin{array}{c}1987 \\
\text { gestantes }\end{array}$ & $\begin{array}{l}\text { Determinar a } \\
\text { prevalência de } \\
\text { sintomas } \\
\text { ansiosos e } \\
\text { depressivos em } \\
\text { gestantes } \\
\text { durante a } \\
\text { pandemia de } \\
\text { COVID-19 e } \\
\text { identificar } \\
\text { fatores de } \\
\text { proteção. }\end{array}$ & $\begin{array}{l}\text { Observou-se elevação dos } \\
\text { sintomas de ansiedade } \\
\text { (57\%) e depressão (37\%) } \\
\text { em comparação com } \\
\text { coorte de gravidez pré- } \\
\text { pandêmica. Melhor } \\
\text { suporte social e prática de } \\
\text { atividade física foram } \\
\text { associados a menores } \\
\text { sintomas de ansiedade e } \\
\text { depressão. }\end{array}$ & $\begin{array}{l}\text { Fatores que exacerbam a } \\
\text { ansiedade e depressão são } \\
\text { preocupações sobre não } \\
\text { receber os cuidados pré- } \\
\text { natais adequados, tensão } \\
\text { no relacionamento e } \\
\text { isolamento social. }\end{array}$ \\
\hline $\begin{array}{l}\text { Zhou et al } \\
\quad(2020)^{1}\end{array}$ & $\begin{array}{c}\text { Estudo } \\
\text { Transversal }\end{array}$ & $3 B$ & $\begin{array}{c}859 \\
\text { mulheres } \\
(544 \\
\text { gestantes } \\
\text { e 315 não } \\
\text { gestantes })\end{array}$ & $\begin{array}{c}\text { Avaliar a } \\
\text { prevalência de } \\
\text { sintomas } \\
\text { psiquiátricos } \\
\text { (depressão, } \\
\text { ansiedade, } \\
\text { desconforto } \\
\text { físico, insônia, } \\
\text { transtorno do } \\
\text { estresse pós } \\
\text { traumático) em } \\
\text { gestantes e } \\
\text { compará-las } \\
\text { com não } \\
\text { gestantes. }\end{array}$ & $\begin{array}{l}\text { Concluiu-se que gestantes } \\
\text { tinham menos sintomas } \\
\text { psiquiátricos do que não } \\
\text { gestantes. Analisados os } \\
\text { fatores sociodemográficos } \\
\text { e os escores das doenças } \\
\text { psiquiátricas não houve } \\
\text { diferença significativa, } \\
\text { exceto para o escore de } \\
\text { sintomas de somatização } \\
\text { entre pacientes com } \\
\text { diferentes níveis de } \\
\text { escolaridade e ocupações. }\end{array}$ & $\begin{array}{l}\text { O período da pesquisa } \\
\text { correspondeu ao estágio de } \\
\text { redução após o ponto } \\
\text { máximo do surto epidêmico } \\
\text { de COVID-19 na China, } \\
\text { aumento de pessoas } \\
\text { curadas e divulgação } \\
\text { dessas informações ao } \\
\text { público. As não gestantes } \\
\text { tinham uma proporção } \\
\text { maior de fumantes, etilistas } \\
\text { e de baixa renda familiar do } \\
\text { que as gestantes. }\end{array}$ \\
\hline
\end{tabular}




\begin{tabular}{|c|c|c|c|c|c|c|}
\hline $\begin{array}{l}\text { Salehi et } \\
\text { al }(2020)^{5}\end{array}$ & $\begin{array}{c}\text { Estudo } \\
\text { Transversal }\end{array}$ & $3 B$ & $\begin{array}{c}222 \\
\text { gestantes }\end{array}$ & $\begin{array}{c}\text { Avaliar a } \\
\text { relação entre o } \\
\text { medo e a } \\
\text { ansiedade } \\
\text { causados pela } \\
\text { COVID-19 em } \\
\text { gestantes e } \\
\text { seus reflexos } \\
\text { na saúde } \\
\text { mental. }\end{array}$ & $\begin{array}{c}\text { Evidenciou-se que a } \\
\text { ansiedade causada pelo } \\
\text { coronavírus tem impacto } \\
\text { direto na saúde mental, e } \\
\text { que uma das principais } \\
\text { causas da ansiedade é o } \\
\text { medo de se infectar e } \\
\text { infectar entes queridos. }\end{array}$ & $\begin{array}{c}\text { Observou-se ainda que a } \\
\text { felicidade ocasionada pela } \\
\text { gravidez tem efeito benéfico } \\
\text { para saúde mental } \\
\text { materna. }\end{array}$ \\
\hline $\begin{array}{c}\text { Dong et al } \\
(2021)^{15}\end{array}$ & $\begin{array}{c}\text { Estudo } \\
\text { Transversal }\end{array}$ & $3 B$ & $\begin{array}{c}156 \\
\text { gestantes }\end{array}$ & $\begin{array}{l}\text { Avaliar ansied } \\
\text { ade e } \\
\text { depressão em } \\
\text { gestantes na } \\
\text { china e seus } \\
\text { fatores de } \\
\text { influência } \\
\text { durante a } \\
\text { pandemia por } \\
\text { COVID-19. }\end{array}$ & $\begin{array}{l}\text { Os níveis de ansiedade } \\
\text { das gestantes } \\
\text { permaneceu o mesmo } \\
\text { quando comparado com } \\
\text { os de gestantes antes da } \\
\text { pandemia. O nível de } \\
\text { depressão foi } \\
\text { significativamente maior. }\end{array}$ & $\begin{array}{l}\text { Na época da aplicação da } \\
\text { pesquisa era noticiado na } \\
\text { China uma fase estável da } \\
\text { pandemia, com número de } \\
\text { curados aumentando, } \\
\text { número de recém- } \\
\text { diagnosticados e crescia na } \\
\text { mídia notícias sobre o } \\
\text { sucesso do parto de } \\
\text { mulheres grávidas } \\
\text { Entretanto, o isolamento e } \\
\text { limitação de acesso a } \\
\text { serviços permanecia. }\end{array}$ \\
\hline $\begin{array}{c}\text { Stepowicz } \\
\text { et al } \\
(2020)^{21}\end{array}$ & $\begin{array}{c}\text { Estudo } \\
\text { Transversal }\end{array}$ & $3 B$ & $\begin{array}{c}210 \\
\text { mulheres } \\
(164 \\
\text { gestantes } \\
\text { e } 46 \\
\text { mulheres } \\
\text { no pós- } \\
\text { parto) }\end{array}$ & $\begin{array}{c}\text { Analisar níveis } \\
\text { de estresse e } \\
\text { ansiedade } \\
\text { experienciados } \\
\text { por gestantes e } \\
\text { puérperas } \\
\text { durante a } \\
\text { pandemia de } \\
\text { COVID-19, } \\
\text { bem como } \\
\text { indicar } \\
\text { condições que } \\
\text { podem } \\
\text { contribuir para } \\
\text { o estresse e a } \\
\text { ansiedade. }\end{array}$ & $\begin{array}{c}\text { O estudo revelou que os } \\
\text { níveis de estresse e } \\
\text { ansiedade em gestantes e } \\
\text { no pós-parto durante a } \\
\text { pandemia de COVID-19 } \\
\text { são significativamente } \\
\text { elevados. }\end{array}$ & $\begin{array}{c}\text { Fatores como idade, } \\
\text { educação, paridade, história } \\
\text { obstétrica agitada, } \\
\text { comorbidades, e o número } \\
\text { de internações mostrou-se } \\
\text { estatisticamente } \\
\text { insignificante na análise. } \\
\text { Parte significativa das } \\
\text { participantes apresentaram, } \\
\text { gestações complicadas, } \\
\text { portanto, podem estar mais } \\
\text { propensas a apresentar } \\
\text { níveis mais elevados de } \\
\text { ansiedade devido a } \\
\text { problemas de saúde } \\
\text { relacionados à gravidez. }\end{array}$ \\
\hline $\begin{array}{c}\text { Hocaoglu } \\
\text { et al } \\
(2020)^{16}\end{array}$ & $\begin{array}{c}\text { Estudo } \\
\text { Transversal }\end{array}$ & 3B & $\begin{array}{c}283 \\
\text { gestantes }\end{array}$ & $\begin{array}{c}\text { Avaliar } \\
\text { sintomas de } \\
\text { ansiedade e } \\
\text { TEPT em } \\
\text { gestantes } \\
\text { durante a } \\
\text { pandemia de } \\
\text { COVID-19. }\end{array}$ & $\begin{array}{c}\text { Mulheres grávidas } \\
\text { apresentaram altos níveis } \\
\text { de ansiedade e sintomas } \\
\text { de TEPT durante a fase } \\
\text { da pandemia de COVID- } \\
19 .\end{array}$ & $\begin{array}{c}\text { Complicações na } \\
\text { gravidez e situação de } \\
\text { emprego do marido foram } \\
\text { os melhores preditores do } \\
\text { estado de ansiedade. Além } \\
\text { disso, a presença de } \\
\text { sintomas relacionados ao } \\
\text { COVID-19 e a } \\
\text { escolaridade predizem os } \\
\text { sintomas de TEPT. }\end{array}$ \\
\hline $\begin{array}{l}\text { Saadati et } \\
\text { al }(2021)^{12}\end{array}$ & $\begin{array}{c}\text { Estudo } \\
\text { Transversal }\end{array}$ & $3 B$ & $\begin{array}{c}300 \\
\text { gestantes } \\
(100 \mathrm{em} \\
\text { cada } \\
\text { trimestre })\end{array}$ & $\begin{array}{c}\text { Avaliar a } \\
\text { ansiedade com } \\
\text { a saúde em } \\
\text { gestantes e } \\
\text { seus fatores } \\
\text { relacionados } \\
\text { durante a } \\
\text { pandemia de } \\
\text { COVID-19. }\end{array}$ & $\begin{array}{c}\text { Observou-se uma } \\
\text { elevação nos níveis de } \\
\text { ansiedade com o decorrer } \\
\text { da gestação, sendo os } \\
\text { escores médio de } \\
\text { ansiedade } 22,3 \pm 9,5,24,6 \\
\pm 9,3 \text { e } 25,4 \pm 10,6 \text { no } \\
\text { primeiro, segundo e } \\
\text { terceiro trimestres } \\
\text { respectivamente. }\end{array}$ & $\begin{array}{c}\text { As participantes não } \\
\text { apresentaram diferença } \\
\text { significativa em relação à } \\
\text { ocupação, escolaridade, } \\
\text { situação econômica e } \\
\text { escolaridade dos cônjuges } \\
\text { nos três trimestres de } \\
\text { gravidez. }\end{array}$ \\
\hline
\end{tabular}




\begin{tabular}{|c|c|c|c|c|c|c|}
\hline $\begin{array}{l}\text { Whitaker et } \\
\text { al }(2021)^{6}\end{array}$ & $\begin{array}{c}\text { Estudo } \\
\text { Transversal }\end{array}$ & $3 B$ & $\begin{array}{c}706 \\
\text { gestantes }\end{array}$ & $\begin{array}{l}\text { Examinar as } \\
\text { mudanças } \\
\text { comportamentai } \\
\text { s de estilo de } \\
\text { vida no início da } \\
\text { pandemia de } \\
\text { COVID-19 entre } \\
\text { gestantes. }\end{array}$ & $\begin{array}{l}17 \% \text { das mulheres } \\
\text { relataram que suas dietas } \\
\text { pioraram. Quanto à } \\
\text { atividade física, } 22 \% \\
\text { relataram que pararam de } \\
\text { ser ativas, } 2 \% \text { relataram } \\
\text { que se tornaram ativas e } \\
76 \% \text { relataram nenhuma } \\
\text { mudança. Quase um terço } \\
\text { das participantes relatou } \\
\text { dormir menos. }\end{array}$ & $\begin{array}{c}\text { Os fatores mais } \\
\text { consistentemente associados } \\
\text { a mudanças adversas durante } \\
\text { a pandemia em todos os três } \\
\text { comportamentos de estilo de } \\
\text { vida foram a presença de } \\
\text { complicações na gravidez, } \\
\text { perda de renda e alterações } \\
\text { nas conexões sociais. }\end{array}$ \\
\hline $\begin{array}{c}\text { Mortazavi; } \\
\text { Mehrabadi; } \\
\text { KiaeeTabar } \\
,(2021)^{10}\end{array}$ & $\begin{array}{c}\text { Estudo } \\
\text { Transversal }\end{array}$ & $3 B$ & $\begin{array}{c}484 \\
\text { gestantes }\end{array}$ & $\begin{array}{l}\text { Investigar o } \\
\text { bem-estar de } \\
\text { mulheres } \\
\text { grávidas e seus } \\
\text { fatores } \\
\text { associados } \\
\text { durante a } \\
\text { pandemia de } \\
\text { COVID-19. }\end{array}$ & $\begin{array}{l}\text { Observaram-se como } \\
\text { preditores de baixo nível de } \\
\text { bem-estar em gestantes a } \\
\text { preocupação com sua } \\
\text { própria saúde, } \\
\text { preocupações com a saúde } \\
\text { do feto e ter pelo menos } \\
\text { uma pessoa infectada com } \\
\text { COVID-19 entre parentes. }\end{array}$ & $\begin{array}{l}\text { Por ser uma pesquisa online, } \\
\text { mulheres de menor poder } \\
\text { aquisitivo podem ter sido } \\
\text { excluídas. Outra provável } \\
\text { limitação é que os } \\
\text { participantes que estavam } \\
\text { mais preocupados tinham } \\
\text { maior probabilidade de não } \\
\text { participar do estudo. Essas } \\
\text { limitações podem ter levado a } \\
\text { observação de menor nível de } \\
\text { preocupação e maior bem- } \\
\text { estar. }\end{array}$ \\
\hline $\begin{array}{l}\text { Yang et al } \\
(2021)^{13}\end{array}$ & $\begin{array}{c}\text { Estudo } \\
\text { Transversal }\end{array}$ & $3 B$ & $\begin{array}{c}19.515 \\
\text { gestantes }\end{array}$ & $\begin{array}{l}\text { Investigar os } \\
\text { efeitos do } \\
\text { bloqueio e } \\
\text { quarentena } \\
\text { obrigatória em } \\
\text { problemas de } \\
\text { saúde mental, } \\
\text { os efeito do } \\
\text { suporte social } \\
\text { percebido e } \\
\text { cognição } \\
\text { desadaptativa e } \\
\text { os efeitos de } \\
\text { moderação da } \\
\text { eSaúde } \\
\text { relacionados em } \\
\text { gestantes. }\end{array}$ & $\begin{array}{l}\text { A quarentena foi fortemente } \\
\text { associada direta e } \\
\text { indiretamente com baixo } \\
\text { estado de saúde mental das } \\
\text { gestantes por meio da } \\
\text { diminuição do suporte social } \\
\text { percebido e aumento da } \\
\text { cognição desadaptativa. O } \\
\text { bloqueio foi indiretamente } \\
\text { associado à saúde mental } \\
\text { devido ao aumento do } \\
\text { suporte social percebido e } \\
\text { cognição desadaptativa. O } \\
\text { uso das redes } \\
\text { sociais fortaleceu o efeito } \\
\text { protetor do suporte social } \\
\text { percebido na saúde mental. }\end{array}$ & $\begin{array}{l}\text { A amostra não foi selecionada } \\
\text { aleatoriamente o que pode } \\
\text { ter introduzido viés de } \\
\text { seleção, e a generalização } \\
\text { dos resultados do estudo para } \\
\text { outras populações deve ser } \\
\text { feita com cautela. }\end{array}$ \\
\hline $\begin{array}{l}\text { Zhang et al } \\
\left(_{(2021)^{2}}\right.\end{array}$ & $\begin{array}{c}\text { Estudo de } \\
\text { Coorte } \\
\text { Prospectivo }\end{array}$ & $2 \mathrm{~B}$ & $\begin{array}{c}456 \\
\text { gestantes }\end{array}$ & $\begin{array}{c}\quad \text { Avaliar a } \\
\text { associação } \\
\text { entre exposição } \\
\text { a notícias sobre } \\
\text { COVID-19, } \\
\text { estilos de vida } \\
\text { pouco } \\
\text { saudáveis e o } \\
\text { risco de aborto } \\
\text { espontâneo } \\
\text { entre gestantes } \\
\text { no estágio inicial } \\
\text { da pandemia. }\end{array}$ & $\begin{array}{c}\text { Observou-se que, em } \\
\text { comparação com o grupo } \\
\text { de uso de mídia de 0,5-2 } \\
\text { horas, o risco de aborto } \\
\text { espontâneo foi } \\
\text { significativamente maior no } \\
\text { grupo de uso de mídia de 2- } \\
3 \text { horas e no grupo de uso } \\
\text { de mídia > } 3 \text { horas. }\end{array}$ & $\begin{array}{l}\text { Mulheres com uso excessivo } \\
\text { de mídia (> } 3 \text { horas) eram } \\
\text { mais propensas a não realizar } \\
\text { atividades físicas, ter } \\
\text { inadequada diversidade } \\
\text { alimentar e sono de má } \\
\text { qualidade. Como limitações } \\
\text { os fatores genéticos e } \\
\text { psicológicos associados ao } \\
\text { aborto espontâneo não foram } \\
\text { investigados. }\end{array}$ \\
\hline $\begin{array}{l}\text { Ostacoli et } \\
a^{2}(2020)^{23}\end{array}$ & $\begin{array}{c}\text { Estudo } \\
\text { Transversal }\end{array}$ & 4 & $\begin{array}{c}163 \\
\text { gestantes }\end{array}$ & $\begin{array}{c}\text { Avaliar a } \\
\text { prevalência e os } \\
\text { fatores } \\
\text { associados de } \\
\text { depressão pós- } \\
\text { parto e TEPT } \\
\text { durante a } \\
\text { pandemia de } \\
\text { Covid-19. }\end{array}$ & $\begin{array}{l}\text { A prevalência de sintomas } \\
\text { depressivos e de estresse } \\
\text { pós-traumático no pós-parto } \\
\text { entre as gestantes que } \\
\text { deram à luz durante a } \\
\text { pandemia de Covid-19 foi } \\
\text { maior do que a relatada em } \\
\text { estudos pré-pandêmicos. }\end{array}$ & $\begin{array}{l}\text { Especulou-se que o aumento } \\
\text { dos sintomas depressivos e } \\
\text { pós-traumáticos encontrados } \\
\text { pode estar relacionado mais } \\
\text { ao estado geral de } \\
\text { preocupação com a pandemia } \\
\text { do que a fatores específicos } \\
\text { com impacto direto na } \\
\text { experiência do parto. }\end{array}$ \\
\hline
\end{tabular}




\begin{tabular}{|c|c|c|c|c|c|c|}
\hline $\begin{array}{l}\text { Karavadra } \\
\quad \text { et al } \\
(2020)^{20}\end{array}$ & $\begin{array}{c}\text { Estudo } \\
\text { Transversal }\end{array}$ & $2 \mathrm{~B}$ & $\begin{array}{l}1451 \\
\text { mulheres } \\
\text { (1221 no } \\
\text { pré-natal e } \\
230 \text { no } \\
\text { pós-parto) }\end{array}$ & $\begin{array}{l}\text { Explorar as } \\
\text { percepções de } \\
\text { gestantes sobre } \\
\text { COVID-19 e } \\
\text { suas } \\
\text { experiências de } \\
\text { saúde. }\end{array}$ & $\begin{array}{l}\text { As gestantes sentiam-se } \\
\text { preocupadas quanto a } \\
\text { transmissão do } \\
\text { coronavírus, mudanças no } \\
\text { cuidado pré-natal, dúvida } \\
\text { da presença de um } \\
\text { parceiro de parto e a } \\
\text { maneira como as } \\
\text { informações são } \\
\text { comunicadas sobre as } \\
\text { mudanças nos serviços. }\end{array}$ & $\begin{array}{c}\text { Por ser um estudo online os } \\
\text { pesquisadores não puderam } \\
\text { fazer mais perguntas para } \\
\text { esclarecer as respostas, a } \\
\text { pesquisa atingiu aqueles } \\
\text { com acesso online, portanto, } \\
\text { resultando em viés de } \\
\text { resposta. }\end{array}$ \\
\hline $\begin{array}{l}\text { Souza et } \\
\text { al }(2020)^{3}\end{array}$ & $\begin{array}{l}\text { Relato de } \\
\text { Experiência }\end{array}$ & 4 & $\begin{array}{c}100 \\
\text { gestantes }\end{array}$ & $\begin{array}{c}\text { Relatar a } \\
\text { vivência de um } \\
\text { círculo de } \\
\text { cultura virtual, } \\
\text { sobre o } \\
\text { enfrentamento } \\
\text { da CoVid-19 e } \\
\text { as } \\
\text { repercussões } \\
\text { para a saúde } \\
\text { das gestantes. }\end{array}$ & $\begin{array}{l}\text { Foi possível por meio dos } \\
\text { diálogos do grupo aliviar } \\
\text { angústias e medos, além } \\
\text { de ressignificar ações e } \\
\text { criar estratégias de } \\
\text { resiliência. }\end{array}$ & $\begin{array}{c}\text { Este estudo teve como } \\
\text { limitador o acesso a } \\
\text { tecnologias de comunicação. }\end{array}$ \\
\hline
\end{tabular}

Tabela 1: Referências selecionadas a partir das bases de dados destacando os autores do artigo, ano, abordagem metodológica, nível de evidência, tamanho amostral , os principais resultados e observações do estudo.

(Fonte: Autoria própria)

em saúde e, portanto, conhecimento de que infecções virais podem oferecer riscos à gestação (16). Entretanto, pouco se sabe ainda acerca das repercussões físicas da COVID19 nas gestantes e nos fetos, o que gera uma situação de grande alarde para essas mulheres diante do contexto pandêmico.

Portanto, partindo da necessidade de se informar mais sobre a COVID-19, assim como a maioria das pessoas, $\mathrm{Ng}$ et. al. 2020 detectou que grande parte das mulheres grávidas obtêm explicações sobre o vírus através das mídias sociais, como telejornais, jornais impressos e redes sociais principalmente (22). Todavia, esses meios de comunicação proporcionam o acesso a uma quantidade exorbitante de informações, as quais, muitas vezes, não possuem fontes confiáveis (2).

Com isso, há um compartilhamento demasiado de fake news sobre a pandemia de COVID-19, corroborando com a propagação de hábitos incompatíveis com as recomendações médicas e com os protocolos sanitários, como a utilização errônea de máscaras e a automedicação. Além disso, uma vez diante da situação de medo instaurado, essas mulheres se tornam agentes disseminadores de notícias falsas em seu convívio social.

Outrossim, pesquisas comprovaram que a exposição a informações de forma excessiva pode provocar problemas psicológicos, como ansiedade, depressão e estresse (11). Isso acontece, visto que, ao se estar em contato contínuo com notícias desastrosas sobre o aumento do número de casos de COVID-19 e das mortes geradas pela doença, aumenta-se a sensação de medo de se contaminar pelo vírus (2).

Em decorrência dessa preocupação profunda, muitas grávidas relataram terem desenvolvido obsessão pelo cumprimento de protocolos sanitários, abusando do uso de álcool e de hipoclorito de sódio para desinfetar superfícies e alimentos (11). Dessa forma, ao longo do contexto pandêmico, uma parcela significativa das gestantes reduziu drasticamente o consumo de informações e o uso de mídias sociais, a fim de evitar elevar os níveis de estresse e de ansiedade $(2,16)$.

Em contrapartida, pesquisas elucidaram que as mídias sociais também se mostraram como uma excelente ferramenta de apoio para as grávidas, uma vez que permitiram que as gestantes esclarecessem dúvidas diretamente com os profissionais de saúde e, assim, desenvolveram novas formas de combate a fake news $(11,13)$. Ademais, as redes sociais possibilitaram que as mulheres grávidas entrassem em contato com os familiares e interagissem entre si para trocar experiências sobre a gestação, reduzindo os níveis de estresse desencadeados pela pandemia de COVID-19 (2,3).

Além disso, estudos de Whitaker et al. (2021) evidenciaram modificações no estilo de vida das mulheres grávidas, que estão relacionadas à prática de atividades físicas, ao sono e à alimentação (6). Dentre essas mudanças, a principal é que as grávidas reduziram a frequência com que realizam exercícios físicos. Isso ocorreu substancialmente em razão das políticas de isolamento e do distanciamento social impostos pela pandemia de COVID-19, o que privou as gestantes de terem acesso a academias e de realizar atividades físicas com acompanhamento de profissionais de educação física ao ar livre e em domicílio $(2,3,6)$.

Associado a isso, tem-se que as complicações as quais acontecem no processo de gravidez foram um fator determinante para 0 sedentarismo entre as grávidas estudadas, especialmente aquelas que estavam entre $02^{\circ}$ e $03^{0}$ trimestre de gravidez (12). Essa predisposição ao sedentarismo evidencia repercussões significativas, com ênfase ao sistema nervoso, uma vez que há a diminuição 
de liberação de hormônios específicos antiinflamatórios e que proporcionam uma sensação de alívio, bem-estar e relaxamento como a endorfina. Com a redução dessas atividades físicas diárias, confere-se que há uma tendência maior para o aumento de níveis de ansiedade em gestantes $(6,17)$.

Outrossim, as mulheres grávidas possuem naturalmente um sono mais prejudicado se comparado a mulheres nãográvidas, apresentando uma menor quantidade de horas de sono e baixa qualidade dele. Entretanto, as preocupações e estresses provocados pela pandemia de COVID-19, como o medo de alguém da família ser contaminado e de perder o emprego, provocaram um prejuízo ainda maior na qualidade do sono e um aumento dos casos de insônia entre as gestantes $(3,6,19)$.

Ademais, durante esse contexto, Zhang et. al. (2021) percebeu que mulheres grávidas que não praticavam atividades físicas rotineiramente também não possuíam o sono regulado e apresentavam desequilíbrios alimentares 2 . Todavia, uma pesquisa qualitativa realizada no Irã mostrou que, no começo da pandemia de COVID-19, muitas gestantes melhoraram os hábitos alimentares, trocando alimentos industrializados e refeições preparadas em restaurantes por comidas caseiras e pelo consumo de mais legumes e verduras cozidos, o que pode ter ocorrido em razão do medo de se contaminar com o vírus (11).

Assim, vale ressaltar que essas alterações nos comportamentos saudáveis, como a diminuição da prática de atividades físicas, o sono desregulado e a dieta alimentar desequilibrada se mostraram bastante presentes entre as gestantes. Tal estilo de vida não saudável foi apontado por estudos analisados como um fator de risco para a saúde das grávidas e do bebê, podendo provocar casos de ansiedade e de aborto $(2,3,6)$. Desse modo, essas mulheres desenvolvem mais sequelas psicológicas, como TEPT e depressão, em virtude do processo traumático do abortamento indesejado e da frustração das expectativas criadas durante a gestação, deixando-as receosas com os possíveis problemas enfrentados em uma nova gravidez.

Somado aos maus hábitos de vida, sabe-se que o aparecimento de complicações, de malformações e de aborto ao longo da gravidez e do período puerperal podem ser facilitados pela falta de um acompanhamento médico adequado. Partindo desse princípio, percebeu-se que as principais preocupações das mulheres grávidas, nesse contexto, são relacionadas às novas condutas do pré-natal, parto e pós-natal. Isso acontece, pois a grande parte dos ambientes hospitalares, devido aos riscos de infecção por COVID-19, limitaram o número de consultas para acompanhamento gestacional e passaram a utilizar também a telemedicina como uma ferramenta de auxílio médico $(13,18)$.

Apesar dos esforços dos sistemas de saúde para contornar os obstáculos impostos pela pandemia, a maioria das gestantes não se sentem confortáveis nos ambientes hospitalares por medo de contrair a COVID-19 ou de seus bebês se infectarem (3). Consequentemente, desencadeou-se um aumento do adiamento e/ou cancelamento de consultas de verificação médica de rotina, dos partos realizados em domicílio e da percentagem de grávidas ansiosas e aflitas com os potenciais reflexos do abandono dos cuidados hospitalares $(4,18)$. Além disso, a despeito dos serviços online não oferecerem riscos às grávidas, ainda há uma descrença com relação à eficácia desses atendimentos por uma parcela significativa das gestantes, especialmente pela falta de contato humano físico e de ambiente acolhedor, o que prejudicou também a continuação do acompanhamento médico (4).

Dessa maneira, diante dos impasses gerados do cenário vigente da pandemia da COVID-19, presencia-se uma interdependência entre os fatores predisponentes e os resultados obtidos, agravando os mesmos. Ao olhar clínico, compreende-se que há a desregulação entre os neurotransmissores, prevalecendo os inibitórios como o ácido gama-aminobutírico (GABA) e a glicina. Com esse aumento, reflete-se no organismo humano sentimentos de solidão, abandono, aumento de depressão, ideação suicida e pensamentos de automutilação. E para que ocorra esses desníveis Souza et al., (2020) afirma que a história prévia da doença, a gravidez não planejada, a ausência de parceiro ou de suporte social, a condição elevada de estresse percebido, a história passada ou presente de complicações obstétricas, a perda fetal e o problema financeiro são condições decisivas no prognóstico da paciente $(3,14)$.

Todavia, percebe-se que assim como a gestante pode ter sintomatologia inibitória no SNC, obtendo fenômenos os quais desencadeiam uma depressão, poderá haver também o fenômeno inverso, em que há uma descarga de neurotransmissores excitatórios no cérebro. Nesse sentido, patologias associadas como ataques de pânico, estresse, TEPT, ansiedade, além do sentimento de medo de contaminação e da sensação de planos frustrados foram bem evidenciadas, visto que o que era comum antes da pandemia tornou-se abruptamente proibido e impactou a vida dessas mulheres $(15,16)$. Por conseguinte, diversas gestantes tiveram que se realocar ao novo cenário e, com isso, adaptar-se da melhor maneira para a mudança do cenário gestacional (3). Entretanto, pela sobrecarga emocional dessa readequação, houve uma contribuição efetiva para a alteração na qualidade da sanidade mental $(18,23)$.

Dessa forma, as grávidas - quando acometidas ou mesmo com o medo da COVID-19 - apresentam uma preocupação deliberada que, quando agravada, corrobora para um tratamento de longa duração à base de antipsicóticos, benzodiazepínicos, entre outros. Isso ocasiona um alto custo na manutenção das medicações, além de reações adversas importantes que esses remédios trazem consigo e afetam diretamente na qualidade de vida da paciente (16).

Este estudo apresentou algumas limitações, como a qualidade dos artigos, visto que a maioria se adequa ao critério 3B (Tabela 1), não havendo, portanto, uma quantidade significativa de estudos com alto nível de evidência científica. Além disso, por ser uma problemática recente, com menos de dois anos, não há dados suficientes acerca das repercussões psicológicas dela a longo prazo. Assim, para compreender 
melhor isso, novas pesquisas devem ser realizadas para investigar tais consequências, e futuras revisões podem incluir artigos de outros idiomas e bases de dados.

\section{CONCLUSÃO}

Perante o exposto, observa-se que a pandemia da COVID19 ocasiona diversos efeitos negativos para a saúde psiquiátrica da gestante - como ansiedade, aumento do estresse, depressão e sintomatologia de transtorno de estresse pós-traumático - proporcionados pelo isolamento social, modificações nos hábitos de vida com diminuição das práticas saudáveis, escassez de dados fidedignos sobre os impactos do vírus para a gravidez e o feto, uso excessivo de mídias sociais e consumo de informações com fontes duvidosas. Desse modo, destaca-se a necessidade de se realizar modificações nos cuidados em saúde dessa população antes, durante e após o parto frente às adversidades causadas pela pandemia. De forma que sejam sanadas as lacunas na continuidade do atendimento, por meio da elaboração de planos alternativos para serviços e ações de saúde com estratégias para capacitação do profissional em saúde, monitorização das pacientes e desenvolvimento de terapêuticas psicossociais com operacionalização remota que atendam as particularidades de cada gestante.

\section{CONFLITO DE INTERESSE}

Os autores declaram que não houve conflito de interesse

\section{FINANCIAMENTO}

Os autores declaram que não houve financiamento

\section{REFERÊNCIAS}

1. Zhou Y, Shi H, Liu Z, Peng S, Wang R, Qi L, et al. The prevalence of psychiatric symptoms of pregnant and non-pregnant women during the COVID-19 epidemic. Transl Psychiatry [Internet]. 1 de dezembro de 2020 [citado 16 de abril de 2021];10(1). Available at: https://pubmed.ncbi.nlm.nih.gov/32950999/

2. Zhang X, Liu J, Han N, Yin J. Social media use, unhealthy lifestyles, and the risk of miscarriage among pregnant women during the COVID-19 pandemic: Prospective observational study. JMIR Public Heal Surveill [Internet]. 1 de janeiro de 2021 [citado 3 de maio de 2021];7(1). Available at: https://pubmed.ncbi.nlm.nih.gov/33293263

3. Souza JB de, Heidemann ITSB, Vendruscolo C, Pitilin EDB, Maestri E, Madureira VSF. Reflexões sobre o enfrentamento do coronavírus disease 2019: diálogos virtuais com gestantes. Rev Enferm do Centro-Oeste Min. 30 de dezembro de 2020;10
4. Shayganfard M, Mahdavi F, Haghighi M, Bahmani DS, Brand S. Health anxiety predicts postponing or cancelling routine medical health care appointments among women in perinatal stage during the covid-19 lockdown. Int J Environ Res Public Health [Internet]. 1 de novembro de 2020 [citado 3 de maio de 2021];17(21):1-13. Available at: https://pubmed.ncbi.nlm.nih.gov/33182388

5. Salehi L, Rahimzadeh M, Molaei E, Zaheri H, Esmaelzadeh-Saeieh $\mathrm{S}$. The relationship among fear and anxiety of COVID-19, pregnancy experience, and mental health disorder in pregnant women: A structural equation model. Brain Behav [Internet]. 1 de novembro de 2020 [citado 16 de abril de 2021];10(11). Available at: https://pubmed.ncbi.nlm.nih.gov/32969190

6. Whitaker KM, Hung P, Alberg AJ, Hair NL, Liu J. Variations in health behaviors among pregnant women during the COVID-19 pandemic. Midwifery [Internet]. 1 de abril de 2021 [citado 3 de maio de 2021];95. Available at: https://pubmed.ncbi.nlm.nih.gov/33508485

7. Hamzehgardeshi Z, Omidvar S, Amoli AA, Firouzbakht $M$. Pregnancy-related anxiety and its associated factors during COVID-19 pandemic in Iranian pregnant women: a web-based cross-sectional study. BMC Pregnancy Childbirth [Internet]. 1 de dezembro de 2021 [citado 3 de maio de 2021];21(1). Available at: https://pubmed.ncbi.nlm.nih.gov/33722198

8. Botelho LLR, Cunha CC de A, Macedo M. O Método Da Revisão Integrativa Nos Estudos Organizacionais. Gestão e Soc. 2011;5(11):121

9. Oxford Centre for Evidence-based Medicine Levels of Evidence Working Group. The Oxford Levels of Evidence 2: Oxford Centre for Evidence-Based Medicine 2009. Available at: https://www.cebm.net/2009/06/oxford-centre-evidencebased-medicine-levels-evidence-march-2009/ accessed 7th April 2020

10. ortazavi F, Mehrabadi M, KiaeeTabar R. Pregnant women's well-being and worry during the COVID-19 pandemic: a cross-sectional study. BMC Pregnancy Childbirth [Internet]. 1 de dezembro de 2021 [citado 3 de maio de 2021];21(1). Available at: https://pubmed.ncbi.nlm.nih.gov/33451292

11. Mortazavi F, Ghardashi F. The lived experiences of pregnant women during COVID-19 pandemic: a descriptive phenomenological study. BMC Pregnancy Childbirth [Internet]. 1 de dezembro de 2021 [citado 3 de maio de 2021];21(1). Available at: https://pubmed.ncbi.nlm.nih.gov/33685398

12. Saadati $N$, Afshari $P$, Boostani $H$, Beheshtinasab $M$, Abedi $P$, Maraghi E. Health anxiety and related factors among pregnant women during the COVID-19 pandemic: a cross-sectional study from Iran. BMC Psychiatry [Internet]. 1 de dezembro de 2021 [citado 3 de maio de 2021];21(1). Available at: https://pubmed.ncbi.nlm.nih.gov/33588794 
13. Yang $X$, Song $B$, Wu A, Mo PKH, Di J, Wang Q, et al. Social, Cognitive, and eHealth Mechanisms of COVID19-Related Lockdown and Mandatory Quarantine That Potentially Affect the Mental Health of Pregnant Women in China: Cross-Sectional Survey Study [Internet]. 22 de janeiro de 2021[citado 3 de maio de 2021]; 23(1):e24495. Available at: https://pubmed.ncbi.nlm.nih.gov/33302251

14. Wu Y, Zhang C, Liu H, Duan C, Li C, Fan J, et al. Perinatal depressive and anxiety symptoms of pregnant women during the coronavirus disease 2019 outbreak in China. Am J Obstet Gynecol [Internet]. 1 de agosto de 2020 [citado 16 de abril de 2021];223(2):240.e1240.e9. Available

at:

https://pubmed.ncbi.nlm.nih.gov/32437665

15. Dong H, Hu R, Lu C, Huang D, Cui D, Huang G, et al. Investigation on the mental health status of pregnant women in China during the Pandemic of COVID-19. Arch Gynecol Obstet [Internet]. 1 de fevereiro de 2021 [citado 3 de maio de 2021];303(2):463-9. Available at: https://pubmed.ncbi.nlm.nih.gov/33009997

16. Hocaoglu M, Ayaz R, Gunay T, Akin E, Turgut A, Karateke A. ANXIETY AND POST-TRAUMATIC STRESS DISORDER SYMPTOMS IN PREGNANT WOMEN DURING THE COVID-19 PANDEMIC'S DELAY PHASE. Psychiatr Danub. [Internet] 2020;32(4). Available https://hrcak.srce.hr/index.php?show=clanak\&id_clanak jezik=364806

17. Sinaci S, Ozden Tokalioglu E, Ocal D, Atalay A, Yilmaz $\mathrm{G}$, Keskin $\mathrm{HL}$, et al. Does having a high-risk pregnancy influence anxiety level during the COVID-19 pandemic? Eur J Obstet Gynecol Reprod Biol [Internet]. 1 de dezembro de 2020 [citado 3 de maio de 2021];255:190-6. Available at: https://pubmed.ncbi.nlm.nih.gov/33147531

18. Moyer CA, Compton SD, Kaselitz E, Muzik M. Pregnancy-related anxiety during COVID-19: a nationwide survey of 2740 pregnant women. Arch Womens Ment Health [Internet]. 1 de dezembro de 2020 [citado 3 de maio de 2021];23(6):757-65. Available at: https://pubmed.ncbi.nlm.nih.gov/32989598

19. Lebel C, MacKinnon A, Bagshawe M, Tomfohr-Madsen L, Giesbrecht G. Elevated depression and anxiety symptoms among pregnant individuals during the COVID-19 pandemic. J Affect Disord [Internet]. 1 de dezembro de 2020 [citado 16 de abril de 2021];277:513. Available

at:

https://pubmed.ncbi.nlm.nih.gov/32777604

20. Karavadra B, Stockl A, Prosser-Snelling E, Simpson P, Morris E. Women's perceptions of COVID-19 and their healthcare experiences: A qualitative thematic analysis of a national survey of pregnant women in the United Kingdom. BMC Pregnancy Childbirth. [Internet] 2020;20(1):1-8.

Available at:https://bmcpregnancychildbirth.biomedcentral.com/ar ticles/10.1186/s12884-020-03283-2
21. Stepowicz A, Wencka B, Bieńkiewicz J, Horzelski W, Grzesiak M. Stress and anxiety levels in pregnant and post-partum women during the COVID-19 pandemic. Int $\mathrm{J}$ Environ Res Public Health [Internet]. 2 de dezembro de 2020 [citado 3 de maio de 2021];17(24):1-9. Available at: https://pubmed.ncbi.nlm.nih.gov/33348568

22. Ng QJ, Koh KML, Tagore S, Mathur M. Perception and Feelings of Antenatal Women during COVID-19 Pandemic: A Cross-Sectional Survey - PubMed [Internet]. [citado 3 de maio de 2021]. Available at: https://pubmed.ncbi.nlm.nih.gov/33164024

23. Ostacoli L, Cosma S, Bevilacqua F, Berchialla $P$, Bovetti M, Carosso AR, et al. Psychosocial factors associated with postpartum psychological distress during the Covid-19 pandemic: a cross-sectional study. BMC Pregnancy Childbirth [Internet]. 1 de dezembro de 2020 [citado 3 de maio de 2021];20(1). Available at: https://pubmed.ncbi.nlm.nih.gov/33208115/ 